\title{
CLO3D-Based 3D Virtual Fitting Technology of Down Jacket and Simulation Research on Dynamic Effect of Cloth
}

\author{
Shuxian Huang and Li Huang \\ Academy of Art and Design, Jiangxi Institute of Fashion Technology, Nanchang, Jiangxi 330201, China \\ Correspondence should be addressed to Li Huang; hiyuredu@163.com
}

Received 2 January 2022; Revised 18 January 2022; Accepted 19 January 2022; Published 18 February 2022

Academic Editor: Xin Ning

Copyright (C) 2022 Shuxian Huang and Li Huang. This is an open access article distributed under the Creative Commons Attribution License, which permits unrestricted use, distribution, and reproduction in any medium, provided the original work is properly cited.

\begin{abstract}
A 3D virtual fitting system for down jackets based on CLO3D is proposed and implemented in this paper. CLO3D system realizes virtual fitting, including three-dimensional body modeling, parametric punching, three-dimensional virtual sewing fitting, fabric texture attribute setting, and dynamic display, which realizes the design and realization of display. The clothing deformation algorithm based on human input posture is used to deform the clothing model, which presents the physical characteristics of virtual clothing more truly. At the same time, the image acquisition equipment is used to obtain the real-life image, and the clothing image transfer algorithm is used to superimpose the virtual clothes and the real-life image. The position of human joints is obtained by feature extraction technology, and the scaling ratio of the virtual clothes is calculated so that the virtual clothes are closely combined with the real-life image. Compared with similar virtual fitting systems in the industry, the information processing process of this system is more intelligent, the interaction is more natural, and the fitting effect is more realistic. We make every effort to make the virtual fitting system reduce the gap between virtual and reality and tend to be more realistic and popular.
\end{abstract}

\section{Introduction}

Today's society is one of rapid development, fierce competition, and ever-emerging new technologies, as well as one in which the Internet has begun to exert a significant influence across all aspects of social life. Online clothing shopping has become a part of daily consumption thanks to the development of clothing e-commerce and e-commerce platforms [1]. When purchasing clothes online, however, consumers are unable to try on the size, style, fabric, and fit of the garments, resulting in an increasingly serious problem of returns and exchanges [2]. Virtual fitting system research has gotten a lot of attention in recent years. The advantage of virtual fitting over traditional fitting is its convenient and quick interactive fitting mode, which provides users with a new shopping experience and creates a free and fashionable shopping mode [3]. Although the clothing industry has traditionally used the clothing model to determine the appropriate relationship between the clothing version and the human body shape, ready-to-wear specifications are not the same as the specific human body size. It is difficult to find clothes with a "tailor-made" effect in existing ready-to-wear specifications due to the diversity of human body shape and the complexity of human body surface [4]. At present, in the virtual fitting system used in the market, the stereoscopic effect of clothing is not ideal, the immersion of humancomputer interaction experience is poor, only the appearance of clothing can be displayed, the factors such as clothing fabric, texture, and drape effect cannot be well expressed, the scaling coefficient of clothing cannot be automatically changed according to the user's body shape characteristics, and the gesture operation is complicated, so the experience effect is poor [5].

With the development of science and technology and the Internet, consumers' consumption behavior is changing accordingly. With the continuous development of humancomputer interaction technology and e-commerce, virtual fitting room has received extensive attention and research. 
Compared with traditional fitting methods, virtual fitting room is more convenient to operate and more natural to interact with, which can bring users a brand-new experience of buying clothes [6]. The so-called virtual fitting means that users can get the same experience as real fitting through some devices without taking any clothes-changing actions. The emergence of such a system greatly simplifies the link of changing clothes and fitting clothes in the traditional clothing retail industry, reduces the consumption of costs such as site time, manpower, and capital, and greatly facilitates businesses and consumers [7]. Clothing try-on effect is primarily perceived through vision and touch, and clothing fit evaluation is determined by whether or not the human body and clothing meet a set of criteria. Although the virtual fitting system is new, it has not yet reached the point where it can completely replace traditional fitting. However, with the arrival of a new wave of IoT industry [8] revolution, the trend of its widespread use is unavoidable. A few manufacturers are eager to try their hand at developing commercial virtual fitting systems. The three-dimensional clothing fitting system is widely used in a variety of industries and locations, and it has helped businesses and merchants improve their commercial channels, user database management, clothing design template database, invoicing management, and customer relationship management [9]. And enterprises will be able to significantly reduce their management and sales costs, adapt to the fastpaced life of the future, and intelligently provide customers with a variety of fitting environments. As a result, this paper proposes and implements a CLO3D-based 3D virtual fitting system for down jackets.

Virtual fitting design is like an organic combination of art and technology, which carries the artistic effects that people see behind the technology [10]. In recent years, with the emergence and development of virtual fitting technology, 3D virtual fitting technology has gradually matured, which is much more convenient than the traditional $2 \mathrm{D}$ dressing system. CLO3D software is a fitting software from CLO Company of South Korea, which is a strong simulation fitting software with complete functions and simple and quick operation. Many scholars in China have studied its application [3]. The online consumer market on the Internet is gradually replacing the traditional sales model and becoming the main way of shopping. Great changes have also taken place in the way of purchasing clothing. Three-dimensional clothing fitting system represents the progress of science and technology, the progress of human thought, and the continuous improvement of human life taste [11]. The pursuit of personalized clothing, which meets the aesthetic needs of users, can better reflect the body characteristics of users and achieve tailor-made services, which is what every consumer yearns for and is also a development direction of virtual fitting technology in the future. In the past, the technology only showed the appearance of clothes, but the fabric, texture, drape effect, and other factors could not be well expressed, and the scaling coefficient of clothes could not be automatically changed according to the user's body shape characteristics, so the experience effect was poor. This paper presents a 3D virtual fitting system for down jackets based on CLO3D. The virtual fitting prototype system designed in this paper is demonstrated, and the feasibility of this kind of system is verified. The results show that the virtual fitting based on CLO3D virtual fitting technology can give users a realistic fitting experience, and the effect reaches or even exceeds the level of similar products, which has strong application innovation.

\section{Related Work}

A virtual fitting construction model based on augmented reality technology was proposed in literature [12]. The user's try-on results are displayed by accurately superimposing and fusing real human body images and virtual clothes. The majority of fittings proposed in literature [13] are derived from traditional garment CAD systems, which are primarily used to simulate fabric cutting in the garment manufacturing process, but which cannot achieve real-time performance and have an unintuitive effect. An improved mesh deformation algorithm was discovered in literature [14]. The improvement is due to the fact that the model deformation is driven by the human body's contour map, and the model is added to simplify the operation, resulting in a more efficient model deformation algorithm. Literature [15] used the three-dimensional software CLO3D to simulate fabric drape, measure the simulated fabric drape coefficient, and create a regression model of the fabric drape coefficient based on the simulated fabric attribute parameters. A network-based fitting application was proposed in literature [16]. This system is primarily based on two-dimensional images, which have low accuracy and applicability. It is commonly used to promote products in the field of e-commerce. Literature [17] proposed a model deformation algorithm for accurately matching virtual clothes to the user's figure, which achieved good results and overcame the shortcomings of similar systems. Although [18] proposes somatosensory interaction and physical simulation, it is only used to control three-dimensional animation, making it difficult to give the user the sensation of wearing clothes. The drape prediction regression model in literature [19] is based on the CLO3D system, and it uses the simulated fabric attribute parameters in CLO3D as the independent variable and the drape index of the real fabric as the dependent variable. The simulated fabric attribute parameters corresponding to the simulated fabric in CLO3D are obtained by measuring the drape index of the real fabric. By adjusting the attribute parameters of the simulated fabric, the drape effect of the clothing worn on the human body can be accurately simulated. Literature [20] establishes a three-dimensional mannequin and three-dimensional virtual sewing fittings through Fuyi clothing CAD/CAM pattern making and the CLO3D system. Based on the function of the virtual fitting software that can quickly change the texture of the fabric and the physical properties of the fabric, set the fabric texture attributes and quickly change the fabric, quickly generate clothing with different physical characteristics of the fabric, and realize the virtual Hanfu simulation design for personalized display. Literature [21] conducted a research on the design of underwear based on the CLO3D system. 
Literature [22] uses CLO3D software to design wedding dresses. The wedding dress is made by hand, the effect is compared, and the change law of the wedding dress 3D design and the method of optimizing the design are summarized. Literature [23] proposed a more detailed method of deleting clothing model structures based on the CLO3D system. Literature [21] introduced the key technologies involved in the virtual fitting system. Some basic principles and research status of human skeleton skinning technology, clothing deformation simulation technology, and clothing image transfer technology are analyzed. The speech recognition technology in the virtual fitting system is introduced. Literature [24] uses CLO3D software to simulate the drape coefficient measurement test of the fabric. Measure various drape indexes of the virtual fabric, and find the influence of the difference in the warp and weft directions of the virtual fabric attribute parameters on its drape indexes. And establish the regression model of the warp and weft virtual fabric attribute parameters and its drape index. On this basis, the real fabric is selected for verification. Literature [25] is based on the use of CLO3D three-dimensional virtual software, from four aspects of model preparation, sample arrangement, physical parameter exploration, and appearance simulation effect optimization, using the controlled variable method to explore the optimized physical parameters of simulated clothing in order to establish a virtual display of men's clothing.

Based on the previous research, this paper proposes a $3 \mathrm{D}$ virtual fitting system for down jackets based on CLO3D. This makes the fitting system have the function of "tailormade," and the effect is more real. It overcomes the shortcomings of similar products and further improves the practicability of the system. In this paper, the algorithm is based on the model mean coding technology, uses the hidden Markov chain method to find the matching relationship between contour points and human body model, and simplifies the accelerated deformation process through the model so as to generate a three-dimensional human body model consistent with the user's body shape. The system shows the effect after the user tries on the clothes by superimposing the real human image and the virtual clothes. It has strong real-time and dynamic characteristics, and virtual clothes can produce effects similar to those of real clothes, such as swaying, fluttering, and folding, according to users' behaviors. And the dynamic display effect of clothing is better.

\section{Methodology}

3.1. CLO3D. CLO3D software is a strong simulation fitting software with complete functions and simple and quick operation. The physical properties of fabrics are the main factors that determine the appearance of clothing. Physical properties of fabrics in the CLO3D system include StretchWeft, Stretch-Wrap, Shear, Bending-Wept, Bending-Wrap, Buckling-Ratio, Buckling-Stiffness, Internal-Damping, Density, FrictionCoefficient, and Pressure. CLO3D includes template window, avatar window, object window, and attribute window for template drawing [26]. 3D avatar fitting and 2D template drawing can be designed and modified at the same time, and the data are synchronized. When selecting objects in the virtual avatar window or template window, you can select multiple templates or internal graphics in the object window, and then you can adjust the attribute values through the attribute window.

All clothes models and mannequins are expressed in animation form for the presentation of clothes, which is based on 3D special effects animation. When users try on clothes, they can choose a model that is close to their own body shape rather than their own fitting, and the fitting effect can be seen in 360 degrees on the fitting screen. CLO3D system provides three types of mannequins as a good software platform for virtual fitting: male model, female model, and Tong Mo. Users can select from the system's mannequins and adjust the size parameters of each part of the virtual avatar to suit their needs [27]. The model library also has a variety of skin tones, hairstyles, shoes, and other accessories to choose from.

Accurate scanning data can be obtained by using a 3D body scanner, which also uses a series of computer iconography to detect users' images. CLO3D-based fashion design and implementation process can be divided into four stages: structural layout, 3D sewing, fabric filling, and dynamic display. In the early stage, we collected and analyzed the data of down jackets and studied different fabrics, styles, and multilayer structures. Then, the $2 \mathrm{D}$ parametric template and fabric map should be imported into CLO3D software in JPG and DXF formats, respectively, and after a series of works such as CLO3D sample sewing, fabric attribute setting, and rendering, virtual animation is finally made for dynamic display. Figure 1 shows the fitting process of down jacket.

CLO3D is a three-dimensional clothing design CAD system that combines panel drawing and virtual fitting functions, allowing pattern drawing and fitting to be completed through a single interface. CLO3D is a 3D clothing simulation program. It can set up a human model, a 2D model, a 3D clothing virtual sewing, fabric simulation, and a dynamic virtual display. It is simple and straightforward to present a design that most designers cannot express with a three-dimensional effect. To achieve the effect of fitting clothes, the board maker can adjust and modify the two-dimensional and three-dimensional transformation patterns. Virtual sample clothes can be created for clothing companies, saving them a lot of money on production costs. Consumers can create clothes based on their own body shape using virtual fitting and other functions at the same time.

By editing the size of the virtual mannequin, the design version of Fuyi clothing CAD software can be imported into the $2 \mathrm{D}$ window, and the virtual mannequin can be tried on by $3 \mathrm{D}$ mannequin simulation. Design the required fabric texture and physical attributes in the fabric physical attribute window, realize the fabric simulation design and dynamic display function, and carry out fast virtual simulation design of clothing.

As far as fitting based on the CLO3D system is concerned, fitting simulation of women's shirts, dresses, 


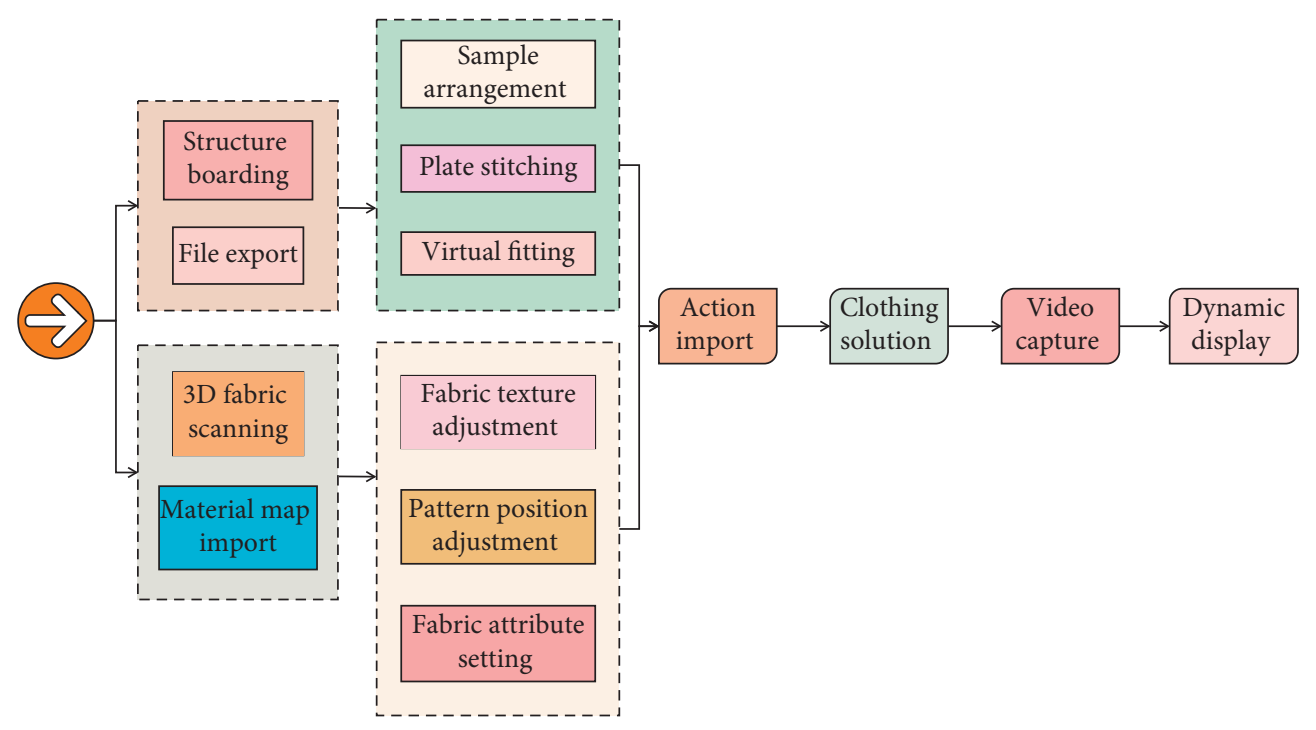

Figure 1: CLO3D down jacket fitting process.

wedding dresses, trousers, Tibetan clothes, men's suits, and underwear has been carried out, and good results have been achieved. Most of the fitting is done from four aspects: model building, sewing fitting, fabric mapping, and simulation display. But there are also some shortcomings. At present, virtual clothes are mostly close-fitting, with common styles and symmetrical structures, which conform to the human body structure. Most of them are suitable for fitting clothes of CLO3D system, but few people have made a breakthrough in style and structure and tried to apply this technology to down jacket styles.

\subsection{Three-Dimensional Virtual Fitting Technology and Dy-} namic Effect of Cloth. The virtual fitting system is to use the principle of computer graphics and the created 3D model of the user's human body to make virtual fitting on the clothing selected by the user, complete the superposition of the clothing pictures to the human body model, and show the wearing effect of the user's virtual fitting [28]. The virtual fitting system mainly consists of four parts: (1) measurement of user's body size, that is, acquisition of three-dimensional body size; (2) creating a mannequin from the user's size; (3) clothing selection operation, that is, selecting the size, position, color, and price range of clothing; (4) user's virtual try-on effect display. The application of 3D virtual technology in textile and garment industry mainly focuses on 3D mannequin, rapid generation of pattern, virtual fitting effect, and dynamic display. It mainly collects data from the human body through three-dimensional human body scanning and inputs the data into the three-virtual simulation system to generate a model consistent with the scanned human body shape. In the system, you can choose clothes to try on technology, and you can clearly see the details, overall effect, and fit of the clothes on the model. You can also already enter more detailed data such as the skin color, hairstyle, and face shape of the customer, which is equivalent to a real customer trying on clothes instead of a virtual model with a rough outline.

In the real world, when a tailor sews clothes, he first cuts the cloth into appropriate shapes according to the components of the clothes, such as sleeves, front piece, and back piece, and then sews the pieces of each part into a complete piece according to the sewing rules of clothes by hand. The operation of virtual fitting mainly includes the following steps: first, the user enters the fitting table, then obtains the three-dimensional data of the mannequin according to the principle of computer graphics, generates the corresponding shell, and determines the corresponding edge positioning lines to facilitate the sewing of ready-made clothes. In the sewing process, due to the requirement of simulated fidelity, the cuffs and collar should be specially treated, and the garment pieces are not allowed to be inserted into the human body. After all the processes are completed, the dressing display can be seen. The specific sewing process of clothing is shown in Figure 2.

Clothing fitting is the process of using computers to simulate customers' real fitting. Mathematical model is the basis of virtual fitting, and image processing is the key to virtual fitting. The system should establish a mathematical model for clothing, realize the fitting process through digital image processing technology, and use various template clothing stored in the computer to try on customers with different shapes and requirements. After the users try on virtual clothing, they should show the fitting effect. At present, users mainly pay attention to the fitting appearance effect, clothing color and fabric effect, and so on. In addition, besides static effect display, it also includes dynamic effect display, for example, whether the dynamic effect of clothing is realistic or not when users are walking and jumping.

The Fuyi garment CAD system is a collection of professional computer programs used in the textile and apparel industries. Pattern design, grading, layout, and printing of pattern and layout drawings of various proportions are all possible with it. The software is simple to use, convenient, 


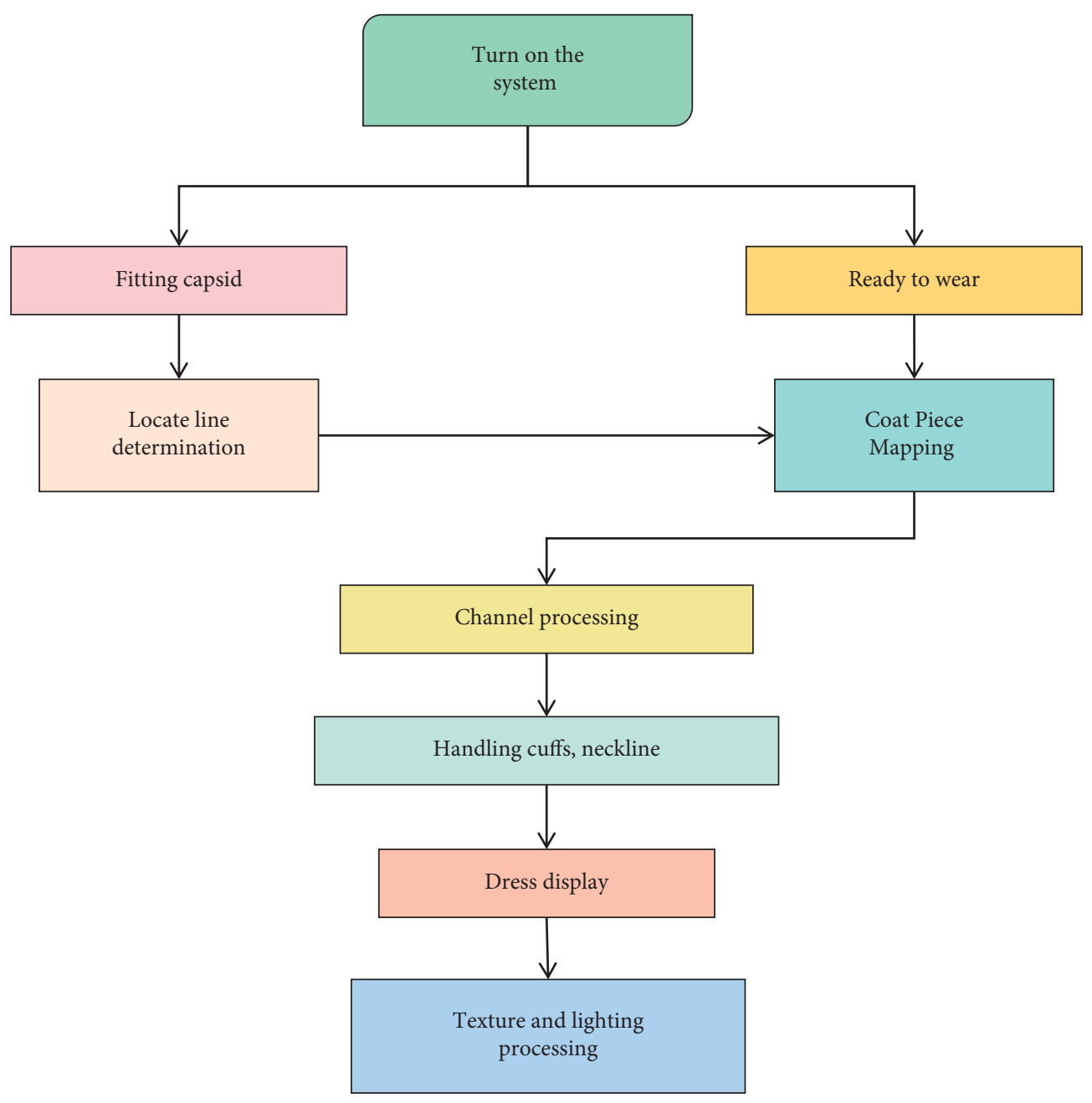

Figure 2: Garment sewing process.

quick, adaptable, and efficient. Formulating and revision are flexible and quick, and they can directly change the size of some parts, as well as the style or specification size, to achieve the effect of changing the pattern or pattern and make it easier to make individual clothing with a single quantity and single cut. The system has an intelligent learning and memorizing function for plate-making methods, processes, and data. The system can automatically complete patterns of various sizes and sizes or samples of a specific human body size, as long as other sizes or individual sizes are input, avoiding the repetitive work of designers and making platemaking work faster and more convenient.

For the model, the system has an arrangement point and an arrangement surface. The garment pieces can be mapped to the corresponding parts using the arrangement point and the arrangement surface, and they can be adjusted, added, or deleted as needed. The three-dimensional arrangement entails placing the templates on the mannequin and the corresponding body parts. The arrangement points are set according to the arrangement surface, and their positions are set according to your requirements. The plates will be placed in the corresponding positions one by one after the arrangement points are placed on the arrangement surface and set at all parts of the virtual model's body at the same time. Sew the samples together in the appropriate order when sewing. The three-dimensional model of the human body is created by entering three-dimensional data about the human body or measuring the size of the human body with a threedimensional measuring instrument. The computer calculates using various algorithms based on this human body information, which necessitates a large amount of data to be stored and a complicated calculation, resulting in high computer performance and storage requirements.

3.3. Three-Dimensional Virtual Fitting Model Is Established. Three-dimensional scanning of human body is the main feature of modern human body measurement technology. It is a high-tech technology based on modern optics, integrating optoelectronics, computer graphics, information processing, computer vision, and other technologies. Build a mannequin, complete the mannequin, build a virtual reallife model, create a tailor-made effect, and realize the dynamic and static simulation of three-dimensional human dress. Parameterized printing is adopted, the style or size of clothing is selected and modified through real-time 
interaction, and the clothing is personalized and customized based on the Internet. Secondly, 3D scanning technology can be used for fabrics, and digital material library mapping can be used to solve the difficulty of obtaining materials in $3 \mathrm{D}$ clothing design so that the effect of $3 \mathrm{D}$ design products is better, more vivid, and faster.

With the sustained and rapid development of science and technology, consumers are also pursuing personalized clothing. Based on the CLO3D virtual fitting software, which has the function of presetting fabric attributes, select fabric texture effect filling for each part of down jacket, and set the corresponding fabric physical attribute parameters. If there are different fabrics filled, that is, the attributes of different fabrics are added in the attribute column of fabrics, the designer can set the physical characteristics of fabrics such as color, transparency, and texture strength in the attribute editor and fill down jackets with different attribute fabrics, so as to quickly formulate personalized clothes and save the cost of making ready-to-wear clothes.

At this time, it is possible to obtain simulation results of clothing deformation with various body postures and behaviors, but getting high-quality real-time simulation results of clothing deformation is still extremely difficult. Extremely small and insignificant changes in body posture will result in a large number of folds and complex deformation effects on clothing, with this change in body movements having a nonlinear and nonlocal influence on clothing deformation. Models bring their own unique postures to the system, which they switch between depending on their needs. Simultaneously, the model's related bones can be controlled to adjust various postures, and the joints of human bones can be adjusted. For example, adjusting the arm opening reveals the fabric's drape performance, and crouching on both knees reveals the clothing's relationship with the human body, such as tightness and pressure. The mannequin's modeling work is completed when the required size is adjusted, and the arrangement point is set.

Build a skin model for each sample pose, and calculate the position of the clothing vertex of each sample pose. The skin scheme uses formula (1) to calculate the position of the clothing vertex of its similar pose.

$$
y^{j}=\sum_{b=1}^{N_{B}}\left\{w_{b}^{j}\left(R_{b}^{j, i n} y^{j}+T_{b}^{j, i n}\right)+R_{b}^{j, i n} \tau_{b}^{j} T_{b}^{j, i n}\right\},
$$

where $R_{b}^{j, \text { in }}$ and $T_{b}^{j, \text { in }}$ represent the relative rotation and translation angle of bone $b$ from $P_{j}$ to $P_{i n} . w_{b}^{j}$ and $\tau_{b}^{j}$, respectively, define the bone rotation and translation weights of clothing vertices. $w_{b}^{j}$ is a bone rotation weight selected based on the clothing deformation effect and can generally be regarded as a standard weight selection method for bone deformation space.

The clothing deformation algorithm based on the input pose calculates the clothing deformation shape for each input pose $P$. The system can obtain the clothing deformation of all approximate postures according to formula (2) and obtain the prediction of the clothing deformation of the input posture.

$$
y^{i n}=\sum_{J=1}^{N_{J}} w^{j}\left(p^{i n}\right) y^{j} .
$$

In the formula, $w^{j}\left(p^{i n}\right)$ is the mixed weight of each vertex of the input pose, which is calculated using sensitive distance measurement technology. Finally, by solving the penetration relationship between the human body grid and the clothing grid, the clothing deformed shape is reconstructed. Sensitivity analysis describes how the clothing deformation simulation results change with small changes in the first-order accuracy of the input parameters. In the clothing deformation scheme of this article, when the internal force and external force of the clothing model reach a balance, the clothing deformation can be calculated in a static state. It can be written as the following formula according to the implicit function theorem:

$$
\Delta y_{m} \approx-(\partial F, \partial y)^{-1}\left\{F\left(x+\Delta x_{m}, y\right)-F(x, y)\right\},
$$

where $\Delta x_{m}$ is the change produced by the small changes in the body mesh with respect to the joint $l=[\mathrm{m} / 3]$. Using formula (3), the sensitivity of each vertex of the corresponding clothing model can be calculated for each posture $j$, as shown in formula (4).

$$
S_{m}=\frac{\partial y}{\partial \Theta_{m}} \in R^{3 N}
$$

The sensitivity of clothing can be approximately expressed as in equation (5) according to the change of the posture $j$ joint angle.

$$
y\left(p^{i n}\right)-y^{j} \cong \sum_{m=1}^{3 N_{\Gamma}} S_{m}^{j} \Delta \Theta_{m} .
$$

Here, $p^{i n}$ is an arbitrary input gesture. $\Delta \Theta_{m}$ is the $m$-th joint rotation angle of $\Theta\left(p^{j}, p\right)$ in this posture.

Kinect is used to capture the user's body posture, 3D models are used to render clothes, physical simulation technology is used to simulate the soft body effect of clothes, and mesh model deformation technology is used to generate clothes models that are consistent with the user's body shape and so on. Finally, users have a better fitting experience and achieve the effect close to the real fitting. Correlation coefficient is a statistic that describes the strength and direction of the linear relationship between them. Its value is between 1 and -1 . The larger the absolute value, the closer the relationship between them.

The high-definition experience and details of the texture map of the fabric can be clearly displayed by using 3D scanning technology to create a high-definition texture map of the fabric. The 3D scanner of the fabric demonstrates the professionalism of the product when compared to the texture map of the fabric obtained by ordinary cameras or high-definition mobile phones. It also has seamless texture processing technology, which not only saves designers time and energy in texture mapping but also accurately displays the fabric material of ready-to-wear clothes while increasing efficiency. Given an input gesture, the nearby sample gesture can be determined using sensitivity-based distance measurement, and the garment deformation result can then be 
calculated. Calculate the deformation of simulated mixed clothing with mixed weight based on distance measurements. Slowing down the rate of blending weight change and using a simple scheme to solve the penetration phenomenon between cloth and body surface can improve the quality of clothing deformation effect.

Suppose that the wind affects the movement of cloth, but the movement of cloth does not affect the wind field. According to the method of fluid mechanics, the force exerted by wind on the corresponding three vertices is calculated by calculating the force exerted by wind on each triangular surface in the model. In this way, the wind force on each particle is the resultant force of the force on its triangular surface. According to the law of fluid mechanics, the force exerted on each triangular surface is

$$
F_{w}=-\frac{1}{2} \rho\left|V_{\text {rel }}\right| c_{d} \partial\left(V_{\text {rel }} \cdot N\right) N
$$

where $F_{\mathrm{w}}$ is the density of $\rho$ air. $c_{d}$ is the drag coefficient of the cloth. $\partial$ is the area of the triangle. $N$ is the normal of the triangle. $V_{\text {rel }}$ is the velocity of the triangular surface relative to the wind. In order to simplify the calculation, set $\rho=1$ and $c_{d}=1$. But

$$
F_{w}=-\frac{1}{2}\left|V_{\text {rel }}\right| \partial\left(V_{\text {rel }} \cdot N\right) N
$$

where $V_{\text {rel }}$ can be calculated by $V_{\text {rel }}=V_{\text {surface }}-V_{\text {wind }}$. Among them, $V_{\text {wind }}$ represents the magnitude and direction of the wind in the scene, and $V_{\text {surface }}$ represents the velocity of the triangle surface itself, which is equal to the average velocity of the triangle vertices. From the idea of numerical integration method, we can get

$$
V_{\text {surface }}=\frac{1}{3}\left(\sum_{i}\left(P_{i}^{\prime}-P_{i}\right)\right), \quad i \in(0,1,2),
$$

where $i$ is the vertex of the triangle, $P_{i}$ is the initial time position of the $i$-th vertex of the triangle, and $P_{i}^{\prime}$ is the next moment position of the $i$-th vertex of the triangle. Finally, the calculated force $F_{w}$ is added to the three vertex particles, respectively. After traversing all the triangles in the model, the resultant wind force on each mass point can be calculated as

$$
F=\sum_{i}^{n} F_{w_{i}},
$$

where $n$ is the number of triangular faces where the particle is located and $F_{w_{i}}$ is the wind force on the $i$ th triangular face where the particle is located.

For each layer, the characterization indexes except drape coefficient and ripple number are added, and the regression equations are obtained to improve the accuracy of clothing simulation effect. In the process of finding the regression equation, the stepwise regression analysis method is adopted. The regression equation not only contains the attribute parameters of the two CLO3D systems, bending, and density but also puts other attribute parameters into the equation according to the stepwise regression analysis results.
Through these three improvements, the accuracy of regression equation can be improved, and then the simulation effect of clothing shape can be improved.

Because the model data is made up of vertices and triangular patches made up of vertices in three-dimensional space, vertices do not form any constraints with each other apart from the topological relationship, and there is a discrete and loose relationship between points. The key challenge in the model deformation process is preserving the geometric modeling information of the model surface so that it can show deformation on a large scale while maintaining the structure on a small scale. We must first define and erect the model's skeleton before we can bind it. We should use the real character joints when erecting the mannequin's skeleton. The skeleton's actions are grounded in reality. Except for the function of protecting internal organs, the human body's functions are essentially the same as those of the real body's bones. The human body's skeleton is divided into upper and lower limb joints for definition and erection. The range of motion of each joint has a radius limit.

When the system gets an input gesture, the corresponding clothing deformation will be generated by mixing the deformation of sample clothing. In many cases, the sensitivity-based weighting scheme between the input gesture and the sample gesture is described as a coherent difference that changes smoothly with time. However, for sudden changes in input posture, the weighting scheme may undergo sudden changes, and continuous abrupt changes may occur in the synthesis stage of clothing. This problem can be prevented by introducing damping, which can be obtained by calculating the value of the current time period and the value of the previous time period.

\section{Results Analysis and Discussion}

When the system detects the user's existence via the input device, it starts to capture the user's body shape parameters and make personalized generation of the clothing model according to these parameters. Then, the simulation cycle is started, and the system continuously acquires the user's current posture, that is, the position of each joint point, adjusts the physical shape of clothes in the current posture, and then renders the three-dimensional virtual clothes and outputs them superimposed with the user's image, showing the effect after "try-on." Place the front and back pieces, sleeve pieces, collar pieces, and so on of the sample pieces in proper positions according to the projection of human body. The virtual sewing tool is used for virtual sewing, then the "synchronization" command is executed, and the complete garment piece is put on the human body in the $3 \mathrm{D}$ model window, thus realizing the transformation process from $2 \mathrm{D}$ to $3 \mathrm{D}$.

Combined with the image recognition algorithm, the keyframe clothing images to be matched can be retrieved more quickly and accurately by using the information of human joints as the keywords of clothing image matching. Combining with image processing equipment and using an improved clothing matching algorithm can greatly improve the recognition accuracy. Figure 3 shows the comparison 


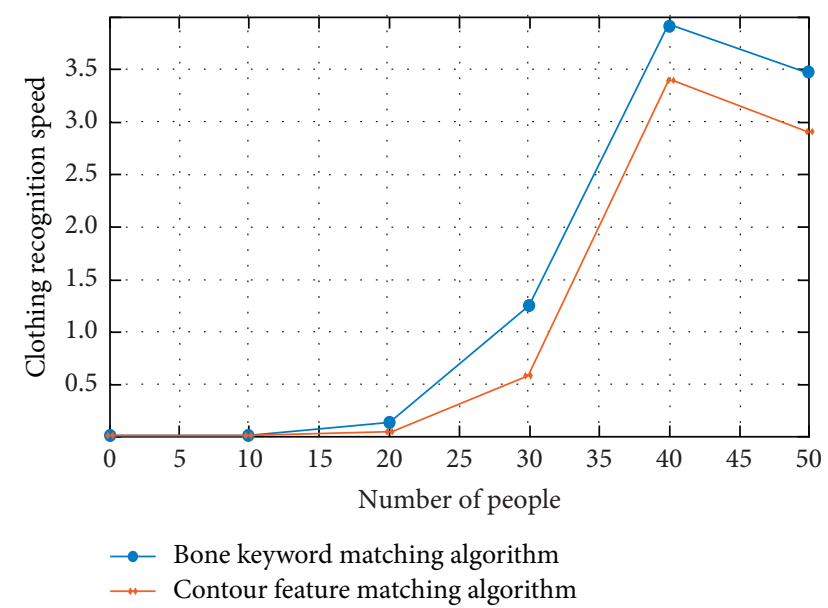

Figure 3: Comparison result of recognition speed of two algorithms.

results of the recognition speeds of the two algorithms. Figure 4 is a comparison result of the accuracy of motion recognition between the two algorithms.

From the experimental data in Figure 4, it can be seen that the clothing matching algorithm using bone keywords can adapt to more users to try on clothes at the same time, the speed of clothing recognition is faster, and the accuracy of motion recognition is also higher. When the deformation of the sampled clothing has little influence on the final result, it may cause distortion of the sample clothing deformation or waste of valuable storage space. For each input system posture, the clothing deformation method based on the input human posture can be used to obtain a realistic clothing deformation effect.

In order to obtain the start time of the effective audio stream and prevent the noise from occupying the processing system, it is necessary to detect the start and end endpoints of the audio stream input into the system by setting the threshold. It is necessary to set at least the low and zerocrossing thresholds of short-term energy in order to distinguish short-term energy from zero-crossing energy. The real starting and ending points of the collected voice stream can be found using a low threshold. It is worth noting that whatever crosses the high threshold is the voice stream's true endpoint. What crosses the low threshold, however, is not necessarily the start and end of the speech, but rather a burst of noise. After determining the fuzzy starting point of speech with a high threshold, the inverse deduction method carefully determines the precise starting point of speech flow with a low threshold, according to the timing index. This algorithm is tested and compared to the standard algorithm. The results are shown in Figure 5.

There are abundant texture details on the surface of real scenery, and people distinguish all kinds of scenery with the same shape according to these texture details. Therefore, the simulation of the texture details of the scene surface plays a very important role in realistic graphics synthesis technology. We call the simulation of the texture details of the scene surface texture mapping technology. Texture mapping technology can be used to simulate the rich texture details of

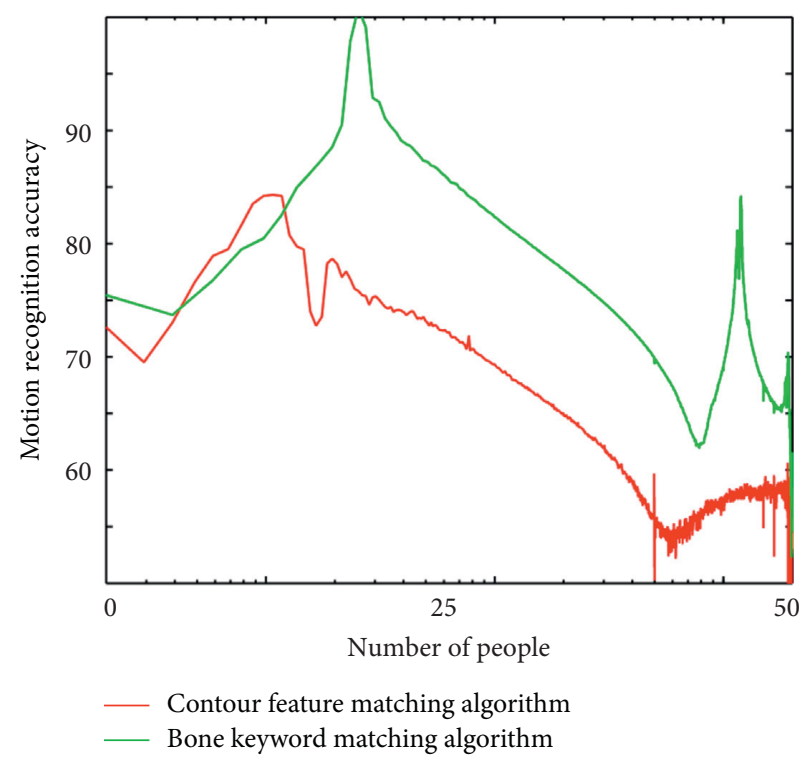

FIGURE 4: Comparison of accuracy of motion recognition between two algorithms.

the scene surface and improve the realism of computergenerated graphics. The error comparison between this algorithm and the traditional algorithm is shown in Figure 6.

It can be seen that the network error after the training of this method is small. Therefore, the algorithm in this paper has certain advantages. Static display can show the details of every part of clothing and show the 360 three-dimensional sense of clothing. In the process of virtual fitting, observe from various angles and clearly observe whether there are any normal wrinkles and other defects such as holes caused by the model's clothes. Static exhibition can also show the pressure diagram, stress diagram, and translucent effect produced by clothing from the virtual person and help users to analyze whether the clothing fits or not through these diagrams to a certain extent. If there is any discomfort, it can be adjusted globally and locally, which is effective and convenient. The results of linear regression analysis are shown in Figure 7.

The comfort of clothing body is presented by pressure distribution and contact point distribution when the pressure test tool is opened from the main menu. When the human body is dressed, the pressure distribution can reflect the stress level of the clothing, which is expressed by color. The larger the red area, the higher the fabric's tensile strength and the greater the clothing pressure. After debugging the clothing, it appears that the red area shrinks and the green area expands, indicating that the fabric stretch and clothing pressure are both weakening. The contact points on the designed clothing have a small and evenly distributed distribution range, indicating that the clothing will not restrict the human body. Figure 8 depicts the dynamic display effect of cloth when compared to the existing methods.

Dynamic display of clothing is the most important part of virtual fitting of clothing, and it is also a bright spot. In the dynamic display of clothing, clothing is naturally the core of the display, and the overall temperament, model 


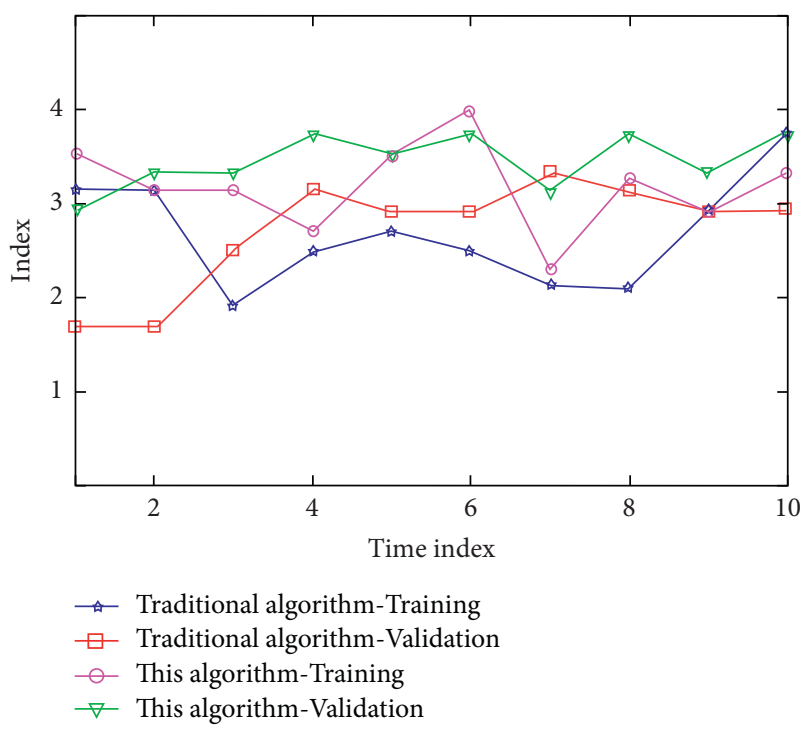

FIGURE 5: Comparison of algorithm training results.

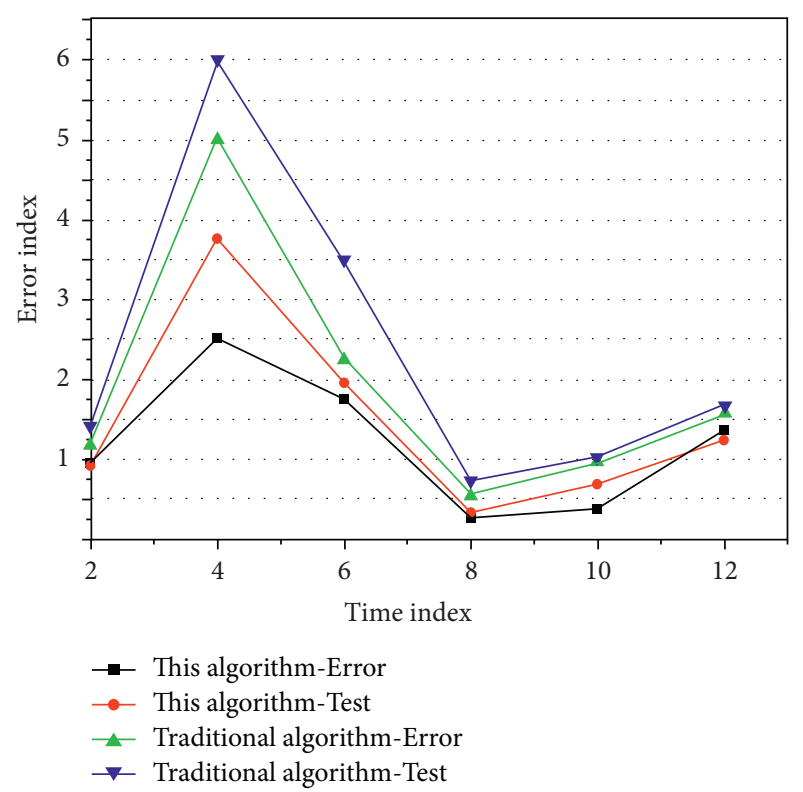

FIGURE 6: Error comparison between this algorithm and traditional algorithm.

performance, and scene display all need to match with the display of clothing. The dynamic display of clothing can not only show the designer's design ideas and design level but also comprehensively observe the overall effect of clothing and the drape of fabrics so that users can better see the style characteristics of the clothing itself. When the wind blows in the human environment, the clothing cloth will swing to a certain extent according to the direction and size of the wind. In this paper, it is also necessary to simulate this situation to simulate the dynamic effect of the clothing cloth under the condition of wind blowing.

Through down jacket ready-to-wear production, static and dynamic display of down jacket is carried out. Compared with the fabric texture details, pleats, and patterns of

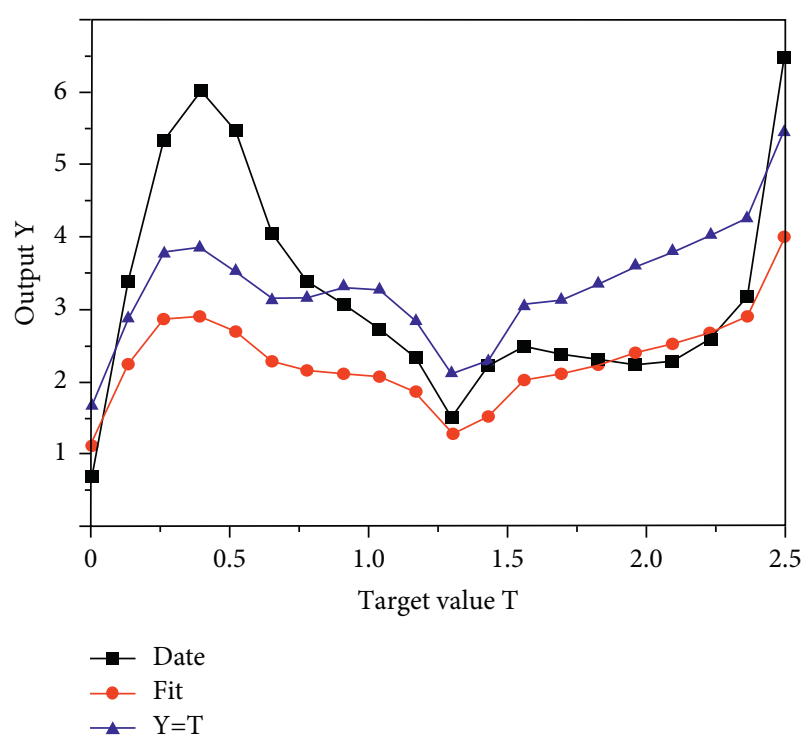

Figure 7: Results of linear regression.

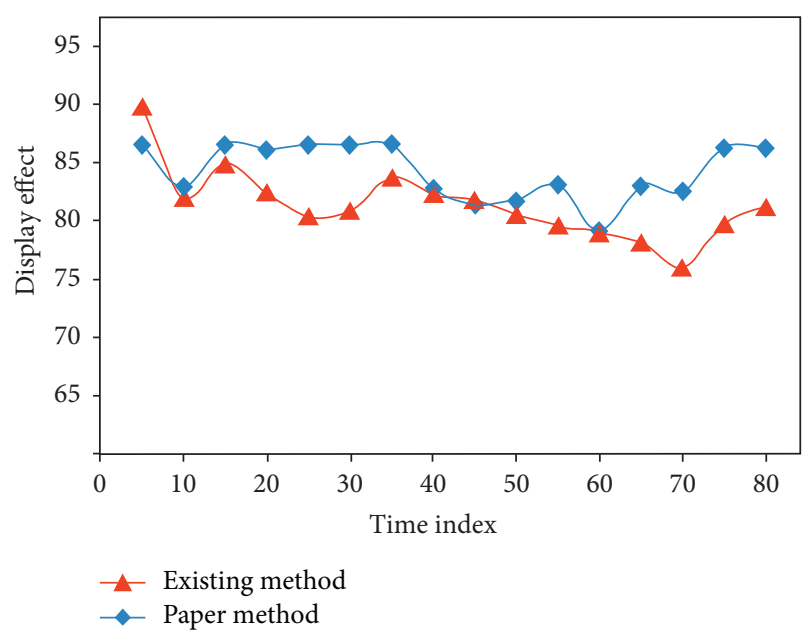

Figure 8: Comparison of dynamic display effect of cloth.

static clothing, the effect is very obvious. The virtual and realistic effects are verified, and the effects of real ready-towear are effectively simulated. The down jacket is evaluated from stress, pressure, translucency, and fitting, and the overall wearing effect, suitability, and comfort of the garment are known through the stress and pressure diagram generated by each part. Experiments are carried out in this chapter, and the experimental results show that the keyframe clothing images to be matched can be retrieved more quickly and accurately by using the information of human joints as the keywords of clothing image matching. Combining with image processing equipment and using an improved clothing matching algorithm can greatly improve the recognition accuracy.

\section{Conclusions}

The research and development of virtual fitting systems have attracted a large number of researchers due to the rapid development of virtual reality technology, but the key 
technologies of virtual fitting are still immature, and some problems have not been well solved. The virtual fitting of down jackets for the CLO3D system is performed in this paper. The key technologies of virtual fitting were studied, and the applications of human skeleton skin technology, clothing deformation algorithm, clothing image transfer algorithm, and voice recognition technology in the virtual fitting system were analyzed, using $3 \mathrm{D}$ virtual fitting as a starting point. The sensitivity analysis method of static balance based on clothing deformation was proposed in the human skeleton skin stage, and a one-way network version framework was proposed to build an optimal skeleton skin solution based on posture, following research and analysis of domestic and foreign achievements in this field. From the dynamic display of the system, we can clearly see the drape effect of clothing during walking and evaluate the effect of real ready-to-wear and virtual fitting. This paper probes into the relationship between the effect of virtual clothing and the subjective feeling of human body dressing. CLO3D system fitting is not only an attempt at down jackets but also a bold attempt at editing and importing mannequins and movements.

\section{Data Availability}

The data used to support the findings of this study are available from the corresponding author upon request.

\section{Conflicts of Interest}

All the authors do not have any possible conflicts of interest.

\section{Acknowledgments}

This work was supported by the 2018 Jiangxi Provincial Department of Education Science and Technology Research Project, "Down Jacket Virtual Simulation Application and Research Based on CLO3D Virtual Fitting Technology" general project, project no. GJJ181036.

\section{References}

[1] L. Liu, R. Wang, Z. Su, X. Luo, and C. Gao, "Mesh-based anisotropic cloth deformation for virtual fitting," Multimedia Tools and Applications, vol. 71, no. 2, pp. 411-433, 2013.

[2] M. L. Nomura and Franzin, "Not enough filling:the extremely expensive down jackets is not as warm as before," China Fiber Inspection, vol. 32, no. 2, pp. 94-98, 2012.

[3] M. Yuan, I. R. Khan, F. Farbiz, and S. S. Yao, "A mixed reality virtual clothes try-on system," IEEE Transactions on Multimedia, vol. 15, no. 8, pp. 1958-1968, 2013.

[4] L. Huang and R. Yang, "Automatic alignment for virtual fitting using 3D garment stretching and human body relocation," The Visual Computer, vol. 32, no. 6, pp. 705-715, 2016.

[5] G. Shi, C. Gao, D. Wang, and Z. Su, "Automatic 3D virtual fitting system based on skeleton driving," The Visual Computer, vol. 37, no. 5, pp. 1075-1088, 2021.

[6] A. Khler, D. Pallarès, and F. Johnsson, "Magnetic tracking of a fuel particle in a fluid-dynamically down-scaled fluidised bed," Fuel Processing Technology, vol. 162, pp. 147-156, 2017.
[7] S. S. Bazyuk, D. S. Kiselev, and Y. A. Kuzma-Kichta, "Thermophysical and corrosion characteristics of the actual and potential fuel-element jackets of light-water reactors in the case of an accident with coolant loss," Journal of Engineering Physics and Thermophysics, vol. 90, no. 1, pp. 1-9, 2017.

[8] J. Kong, C. Yang, J. Wang et al., "Deep-stacking network approach by multisource data mining for hazardous risk identification in Iot-based intelligent food management systems," Computational Intelligence and Neuroscience, vol. 2021, Article ID 1194565, 16 pages, 2021.

[9] Y. He, S. Du, H. Li, Q. Cheng, V. Pavlinek, and P. Saha, " $\mathrm{MnO}_{2}$ /polyaniline hybrid nanostructures on carbon cloth for supercapacitor electrodes," Journal of Solid State Electrochemistry, vol. 20, no. 5, pp. 1459-1467, 2016.

[10] K. Liu, X. Zeng, P. Bruniaux et al., "3D interactive garment pattern-making technology," Computer-Aided Design, vol. 104, pp. 113-124, 2018.

[11] Z. Chang, F. Han, Z. Sun, Z. Gao, and L. Wang, "Three-dimensional dynamic sea surface modeling based on ocean wave spectrum," Acta Oceanologica Sinica, vol. 40, no. 10, pp. 38-48, 2021.

[12] H. He, X. Chen, A. Mehmood et al., "ClothFace: a batteryless RFID-based textile platform for handwriting recognition," Sensors, vol. 20, no. 17, p. 4878, 2020.

[13] N. Rahman, K. G. Tan, and T. A. Rahman, "Modeling of dynamic effect of vegetation for fixed wireless access system," Wireless Personal Communications, vol. 96, no. 1, pp. 1-26, 2017.

[14] L. Jiang, J. Ye, L. Sun, and J. Li, “Transferring and fitting fixed-sized garments onto bodies of various dimensions and postures," Computer-Aided Design, vol. 106, no. 1, pp. 30-42, 2019.

[15] Y. Jiang, R. Guo, F. Ma, and J. Shi, "Cloth simulation for Chinese traditional costumes," Multimedia Tools and Applications, vol. 78, no. 4, pp. 5025-5050, 2019.

[16] A. Seifi, A. Hosseini, M. S. Marefat, and M. S. Zareian, "Improving seismic performance of old-type RC frames using NSM technique and FRP jackets," Engineering Structures, vol. 147, no. 9, pp. 705-723, 2017.

[17] M. Zhang, H. Dong, X. Fan, and R. Dan, "Finite element simulation on clothing pressure and body deformation of the top part of men's socks using curve fitting equations," International Journal of Clothing Science \& Technology, vol. 27, no. 2, pp. 207-220, 2015.

[18] M. Tomoe, "Surface shape features of 3D tight-fitting skirts using angle curvatures in virtual reality," Journal of Textile Engineering, vol. 63, no. 5, pp. 121-129, 2018.

[19] M. F. Hashmi, B. K. K. Ashish, A. G. Keskar, N. D. Bokde, and Z. W. Geem, "FashionFit: analysis of mapping 3D pose and neural body fit for custom virtual try-on," IEEE Access, vol. 8, no. 99, pp. 91603-91615, 2020.

[20] S. J. T. McElroy and G. Thomas, The Papers of the Bibliographical Society of America, The Bibliographical Society of the University of Virginia, Charlottesville, VA, USA, 2011.

[21] B. Broudy, "Jackets that can weather a storm," Popular Science, vol. 288, no. 1, p. 16, 2016.

[22] Y. Chang and H. Zhang, "Study on draping simulation of the fabric with difference in warp and weft in CLO3D," Journal of Beijing Institute of Clothing Technology (Natural Science Edition), vol. 37, no. 2, pp. 33-39, 2017.

[23] R. Dubey and P. Kumar, "Experimental study of the effectiveness of retrofitting RC cylindrical columns using self- 
compacting concrete jackets," Construction and Building Materials, vol. 124, no. 10, pp. 104-117, 2016.

[24] L. Wang, H. Yang, G. Pan, L. Miao, S. Chen, and Y. Song, "Polyaniline-Carbon Nanotubes@Zeolite imidazolate framework-67-carbon cloth hierarchical nanostructures for supercapacitor electrode," Electrochimica Acta, vol. 240, pp. 16-23, 2017.

[25] L. Wang, "Electrothermal regeneration by Joule heat effect on carbon cloth based $\mathrm{MnO}_{2}$ catalyst for long-term formaldehyde removal," Chemical Engineering Journal, vol. 357, pp. 1-10, 2019.

[26] S. A. Cremen and J. P. Mcnulty, "The availability of appropriately fitting personal protective aprons and jackets for angiographic and interventional radiology personnel," $R a$ diography, vol. 20, no. 2, pp. 126-130, 2014.

[27] D. Sukumaran, A. K. Sharma, Y. H. Wasu, P. Pandey, and V. Tyagi, "Knockdown and repellent effect of permethrinimpregnated army uniform cloth against Aedes aegypti after different cycles of washings," Parasitology Research, vol. 113, no. 5, pp. 1739-1747, 2014.

[28] S. R. W. D. F. Channell, A History of Technoscience: Erasing the Boundaries between Science and Technology, Routledge, London, UK, 2017. 